\title{
RELACIÓN ENTRE PROPIEDAD INTELECTUAL, INNOVACIÓNY DESARROLLO: EVIDENCIAS DE DATOS DE PANEL
}

\section{Relationship between intellectual property, innovation and development: Evidences from data panel}

\section{Víctor Gómez-Valenzuela}

Profesor investigador del Instituto Tecnológico de Santo Domingo (INTEC). Avenida Los Próceres No. 49, Los

Jardines del Norte, 10602, Santo Domingo, D. N., República Dominicana. Correo: victor.gomez@intec.edu.do

Recibido: 15/6/2017 • Aprobado: 19/12/2017

\section{Resumen}

En este trabajo se presentan los resultados de un análisis de datos de panel que explora la relación entre propiedad intelectual, innovación y desarrollo. Esta relación es abordada como una oportunidad para superar los desafíos que, en materia de crecimiento y desarrollo, tienen los países de renta media, como la República Dominicana. El panel de datos fue construido a partir de información procedente de distintas fuentes, desde 2005 hasta 2011. El análisis de los datos de panel se realizó en dos momentos: 1) mediante un análisis factorial de componentes principales, para definir los factores de mayor peso en la explicación de la varianza del panel de datos, y 2) utilizando el método de mínimos cuadrados ordinarios, para estimar la relación entre propiedad intelectual, innovación y desarrollo. Los resultados mostrados por los datos de panel destacan que los factores de capital humano, los sistemas de propiedad intelectual, como expresión del grado de desarrollo institucional de las economías, y el esfuerzo en producción de conocimiento e innovación son factores claves en el desempeño y en las dinámicas de crecimiento y desarrollo. Se concluye que el fortalecimiento institucional de los sistemas de propiedad intelectual como pilar de los sistemas de innovación, así como la inversión en ciencia, tecnología e innovación constituyen estrategias viables para promover el crecimiento económico y la salida de la trampa de los ingresos medios en economías como la de República Dominicana.

Palabras clave: propiedad intelectual; innovación; desarrollo.

\section{Abstract}

This paper shows the results of a panel data analysis that explores the relationship between intellectual property, innovation, and development. This relationship is analyzed as an opportunity to overcome the growth and development challenges of a middle-income country such as the Dominican Republic. The data panel was constructed based on information from different sources from 2005 to 2011 . The analysis of the panel data was carried out in two moments: 1) by a factorial analysis of principal components to define the weight of factors in explaining the variance of the data panel; 2) by using the method of ordinary least squares, to estimate the relationship between intellectual property, innovation, and development. The results shown by the panel data highlight those human capital factors, intellectual property systems, as an expression of the degree of institutional strength, the effort in the production of knowledge and innovation are key factors in explaining the performance and dynamics of growth and development. It is concluded that the institutional strengthening of intellectual property systems as a pillar of innovation systems, as well as investment in science, technology, and innovation are viable strategies to promote economic growth and to escape from the trap of middle income in economies such as the Dominican Republic.

Keywords: intellectual property; science and technology; innovation; development. 


\section{Introducción}

El objeto del presente trabajo es analizar la relación entre propiedad intelectual (PI), innovación y desarrollo desde una perspectiva global a partir de la cual extraer aprendizajes que puedan ser útiles para orientar la discusión sobre políticas públicas de ciencia, tecnología e innovación (CTI). Estas reflexiones son particularmente útiles para las relativamente pequeñas economías abiertas y de renta media como la de República Dominicana y, de igual modo, pueden mejorar la comprensión de la importancia de los sistemas de propiedad intelectual como uno de los pilares de los sistemas de innovación (SI). Asimismo, en contextos de grandes asimetrías de capacidades de sistemas nacionales de innovación, como ocurre en América Latina y el Caribe (Dutrénit, 2012), estas reflexiones sobre la importancia de la PI permiten replantear el papel que pueden jugar las políticas de CTI en el cierre de la brecha en materia de crecimiento, desarrollo productivo e inclusión social $\mathrm{Fu}$, Pietrobelli, \& Soete, 2010; Navarro, Benavente, \& Crespi, 2016). ${ }^{1}$

Se ha indicado que la República Dominicana es una economía de renta media, pero ¿qué significa ser una economía de renta media y cómo interactúa esa condición con la relación entre PI, innovación y desarrollo? Lo primero, cómo se verá a lo largo de este trabajo, es que la literatura sobre la relación entre PI, innovación y desarrollo no deja dudas sobre la robustez de esa relación, por lo que

1. En el marco del presente trabajo el desarrollo es comprendido como una dinámica de creación de valor de los sectores productivos, así como de construcción de bienestar e inclusión social. En tal sentido, las dinámicas de desarrollo dependen de manera orgánica y compleja de la retroalimentación positiva de factores como la productividad, la competitividad, la innovación, el bienestar de la población con las capacidades científico-tecnológicas de la sociedad, en la medida en que dichas capacidades están al servicio del bienestar colectivo (Fagerberg \& Srholec, 2008). el aporte de este trabajo no necesariamente supone un grado significativo de novedad al respecto. Ahora bien, como aspecto relevante, la novedad estriba en abordar la relación desde la perspectiva de las economías de renta media, en especial aquellas afectadas por la denominada "trampa de los ingresos medios" (Felipe, 2012), a la que nos referiremos más adelante.

En lo que respecta a lo que significa ser una economía de renta media, siguiendo la "taxonomía" del Banco Mundial, una economía de este tipo es aquella cuyo Ingreso Nacional Bruto (INB o GNI, por sus siglas en inglés) se encuentra entre US\$3,976.00 y US\$ 12,275.00; una economía de renta alta es aquella con un INB superior a US\$12,275.00; y se considera de renta baja cuando se encuentra entre US\$1,006.00 y US\$3,975.00 (Felipe, 2012). Las implicaciones sociales, en términos de desarrollo, que se derivan de los niveles de ingreso de las distintas economías quedan fuera del alcance del presente trabajo. ${ }^{2}$

Ejemplificando lo anterior, se espera que una economía de renta media baja tenga un INB per cápita de entre US\$2,000.00 y US\$7,250.00, y una de renta media alta se espera que tenga un INB per cápita entre US\$7,250 y US\$ 11,750.00 (Felipe, 2012). De acuerdo con el Banco Mundial (World-Bank, 2017), en el año 2015 en el intervalo de economías de renta media alta en América Latina se encontraban países como Argentina (US\$12,450.00), Brasil (US\$9,990.00), Panamá (US\$11,880.00) y Costa Rica (US\$10,400.00). Según la misma fuente, economías como la chilena y la uruguaya son las únicas de América Latina que para el año 2015 se consideraban de altos ingresos, con un INB per cápita de US\$14,100.00 y US\$15,720.00, respectivamente. Aquellas economías con un ingreso nacional bruto

2. Un análisis más detallado y exhaustivo sobre las economías de renta media y sus características económicas y sociales, así como sobre los desafíos en desarrollo que confrontan, puede encontrarse en Nallari, Yusuf, Griffith \& Bhattacharya (2011). 
inferior a US $\$ 2,000.00$ son consideradas de bajos ingresos (Felipe, 2012). ${ }^{3}$

Por consiguiente, en lo que respecta a la relación entre PI, innovación y desarrollo, desde la perspectiva de una economía de renta media, la importancia de esa relación puede ser comprendida desde la ya referida "trampa de los ingresos medios", que consiste en el estancamiento de la dinámica de crecimiento y de aumento de la renta per cápita, como resultado de factores como la reducción de la participación en el empleo de la manufactura, al situarse por debajo del $23 \%$, el declive de la renta per cápita por debajo del $57 \%$ de la frontera tecnológica del país y las dificultades en materia de productividad, competitividad y convergencia tecnológica, entre otros factores (Eichengreen et al., 2013). ${ }^{4}$

De igual modo, la "trampa" ha sido definida en términos operacionales como el número de ańos en que una economía permanece dentro de un determinado intervalo de ingresos, en comparación con el desempeño histórico de aquellas economías que lograron la transición de ingresos medios a altos (Felipe, 2012). De manera que, si una economía determinada permanece en promedio más años en

3. En el caso de la República Dominicana, de acuerdo con el Banco Mundial, el INB per cápita para el ańo 2015 era de US $\$ 6,240.00$, por lo que se encuentra dentro del rango de economía de renta media (World-Bank, 2017). Ahora bien, debe matizarse que dentro de las economías de esta clasificación existen intervalos cuyos umbrales marcan diferencias significativas dentro de las mismas economías clasificadas como de renta media (Eichengreen, Park \& Shin, 2013).

4. Las economías de renta media tienden a caracterizarse por alcanzar unas altas tasas de crecimiento que les permiten avanzar con relativa rapidez (entre dos y tres décadas) de una situación de bajos ingresos per cápita a un escenario de ingresos medios, pero corren el riesgo de quedar atrapadas en una situación de inercia, debido a la desaceleración del crecimiento que se ha denominado en la literatura como "la trampa de los ingresos medios" (Eichengreen et al., 2013). De acuerdo con la evidencia recopilada, para alcanzar un nuevo estado estacionario de ingresos altos, los países de ingresos medios deberían crecer no menos del $3.5 \%$ anual por, aproximadamente, década y media (Felipe, 2012). un determinado intervalo de ingresos, puede argumentarse que se encuentra atrapada en la trampa de ingresos medios.

La evidencia sugiere que aquellas economías cuya población posee mayores niveles de educación secundaria y universitaria, aptitudes tecnológicas y capacidad para "ascender por la escalera tecnológica" (Eichengreen et al., 2013), tienen más posibilidades de evitar la trampa de ingresos medios. Asimismo, la inversión en innovación $y$ en el desarrollo de capacidades tecnológicas constituyen dos estrategias viables de mediano plazo para la salida de la referida trampa (Griffith, 2011). De la misma manera, apuntan que la existencia de estructuras de gobernanza y de políticas públicas de CTI que estimulen el desarrollo y la consolidación de los SI constituyen factores claves, tanto para aprovechar las tendencias tecnológicas globales como para estimular los procesos de cambio tecnológico y convergencia de las economías en desarrollo (Fu et al., 2010; Navarro et al., 2016)s.

Es precisamente en el ámbito de las políticas de CTI y de consolidación de los SI que la relación entre PI, innovación y desarrollo cobra sentido, como herramienta para estimular capacidades de crecimiento endógeno. Tal consolidación permite aprovechar las oportunidades que puedan derivarse de los procesos de apertura y participación en las cadenas globales de valor, lo que dependerá, en términos de propiedad intelectual, del nivel de desarrollo de las economías (Kim, Lee, Park, \& Choo, 2012). Las consideraciones anteriores aportan al análisis de la relación entre PI, innovación y desarrollo una heurística complementaria: la definida a partir de la trampa de los ingresos medios. Esta heurística, aún por construirse y que se sugiere a lo largo de este trabajo, permitirá explorar las implicaciones analíticas, tanto positivas como normativas, que puedan derivarse de la mencionada trampa, con relación al papel de la PI en las dinámicas de desarrollo a largo plazo. 
Desde el punto de vista metodológico, el presente trabajo se sustentó en un abordaje exploratorio que partió primero de una revisión general de la literatura sobre PI, innovación y desarrollo; luego, se articuló con el análisis de datos de panel, el cual fue construido a partir de datos de diversas fuentes entre los años 2005 y 2011. A los fines de simplificar el análisis de los datos de panel, se realizó un análisis factorial por componentes principales. Este enfoque permitió identificar los factores de la PI, la innovación y el desarrollo relevantes desde la perspectiva del trabajo. El abordaje, basado en un análisis exploratorio, constituye una estrategia de uso frecuente en la literatura sobre capital intelectual (Bontis, 1998; Vergauwen, Bollen \& Oirbans, 2007).

\section{Justificación}

El análisis de la relación entre propiedad intelectual y desarrollo es clave para mejorar los procesos de diseño de las políticas públicas relativas a la promoción de los SI, así como de cualquier iniciativa de estímulo al desarrollo de capacidades científico-tecnológicas en la industria, las universidades $o$ en cualquier otro integrante de tales sistemas (Gómez-Valenzuela, 2015; Park, 2002). A nivel de Centro América y el Caribe, si bien se ha avanzado en el desarrollo de políticas científico-tecnológicas y de estímulo a la innovación, aún persiste una brecha importante al considerar como parte integral de estas políticas el reforzamiento de los sistemas de propiedad intelectual (Casalet \& Buenrostro, 2014; Padilla-Pérez \& Gaudin, 2014). En el caso de la República Dominicana, esa brecha no solo persiste, sino que es una marca característica que afecta el desempeño innovador de las firmas dominicanas (Gómez-Valenzuela, 2016).

En lo que respecta a la dinámica de crecimiento, la evidencia empírica demuestra que la República Dominicana, basados en datos del Banco Mundial sobre la clasificación de los niveles de renta del año 2010, se encuentra atrapada en la trampa de los ingresos medios, junto con otras 10 economías de la región, tales como Bolivia, Brasil, Colombia, Ecuador, El Salvador, Guatemala, Jamaica, Panamá, Paraguay y Perú (Felipe, 2012).

\section{Marco analítico general}

Según la Organización Mundial de la Propiedad Intelectual (OMPI), la Propiedad Intelectual (PI) puede definirse como "el conjunto de derechos que corresponden a los autores y a otros titulares respecto de las obras y prestaciones fruto de su creación" (WIPO, 2004). En otras palabras, la PI consiste en las creaciones del intelecto de las personas, operando de manera individual o a través de organizaciones, en sentido amplio: empresas, universidades, centros de investigación, etc. $5^{5}$ se encuentra estrictamente relacionada con la información o con los conocimientos incorporados en objetos tangibles; por consiguiente, no se encuentra en los objetos, sino en el conocimiento contenido y codificado en estos (Arthur, 2009; Dinopoulos \& Kottaridi, 2008).

Dado que los derechos de PI protegen el trabajo y los intereses de los creadores al reconocerles prerrogativas sobre sus obras, en sentido amplio, la PI se refiere a los derechos legales derivados de la actividad intelectual en los distintos campos de la industria, el arte y las ciencias. No obstante, el análisis de la PI va mucho más allá del ámbito legal.

5. La PI cubre dos grandes ramas: los derechos de autor y la propiedad industrial. La primera se refiere a las creaciones artísticas y literarias, y la segunda a las invenciones y los diseños industriales (WIPO, 2004). En la propiedad industrial se incluyen las patentes, los modelos de utilidad, los diseńos industriales, los secretos industriales y las marcas; es precisamente esta rama la que es objeto de análisis en el presente artículo. De manera que, en lo adelante, se hará referencia a la rama de la propiedad industrial al utilizar las siglas PI. 
Desde hace poco más de dos décadas, comenzó a evidenciarse que los sistemas de protección de la PI "influyen" a nivel agregado en los incentivos que tienen los individuos y las firmas para innovar y, por extensión, pueden afectar las dinámicas de crecimiento económico (Dinopolus \& Segerstrom, 2010; Gould \& Gruben, 1996; Higino Shneider, 2005).

Adicionalmente, los sistemas de PI constituyen una parte sustancial del entramado institucional de los SI, los cuales pueden definirse en, sentido general, como el conjunto de instituciones y actores cuyas interacciones permiten la creación de valor a partir de insumos de conocimiento, especialmente las universidades, las empresas y el gobierno (Etzkowitz \& Leydesdorff, 2000). De hecho, para algunos autores, dependiendo del nivel de desarrollo de las distintas economías, los sistemas de PI constituyen por sí mismos el núcleo de los sistemas de innovación, al mediatizar los incentivos para el comportamiento innovador de las firmas (Casper $\&$ van Waarden, 2005; Scotchmer, 2004). Sobre los sistemas de innovación se volverá más adelante, con el fin de comprender su relevancia como marco analítico.

De acuerdo con el Tratado de Ginebra de 1978, los descubrimientos científicos pueden definirse como "el reconocimiento de fenómenos, propiedades o leyes del universo material no conocidos hasta el momento y susceptibles de verificación" (WIPO, 2004). Por tanto, siguiendo ese mismo razonamiento, las invenciones pueden ser consideradas como soluciones a problemas técnicos específicos que se fundamentan en las propiedades o leyes del universo material, las cuales no tienen que ser necesariamente nuevas. Por consiguiente, toda invención descansa en algún tipo de principio derivado de los descubrimientos y conocimientos científicos (Arthur, 2009).

El estímulo para el desarrollo y el aumento del stock del conocimiento científico, a través de la actividad científica y técnica profesional en empresas, universidades, centros de investigación y en los distintos actores de los sistemas de innovación, constituye uno de los elementos centrales de las políticas científicas y tecnológicas que, a su vez, se relacionan con el grado de fortaleza de los sistemas nacionales de protección de la PI (Dinopoulos \& Kottaridi, 2008). Por consiguiente, la evidencia empírica sugiere que la creación de capacidades y competencias científicas y tecnológicas en el capital humano genera diferencias competitivas entre las economías (Cerulli, 2014; Fagerberg \& Srholec, 2008).

De manera que, al pensar en los sistemas de PI y en su articulación con los SI, no es de extrañar el interés que concitan los estudios de los sistemas de propiedad intelectual entre los economistas y entre otros cientistas sociales interesados en el funcionamiento de las políticas públicas de ciencia, tecnología e innovación. Tal interés se intensificó con la entrada en vigencia de la Organización Mundial del Comercio (OMC), en 1995, y del acuerdo global denominado Agreement on trade related aspects of intellectual property rights (Acuerdo sobre aspectos relacionados al comercio de la propiedad intelectual) o TRIPS, por sus siglas en inglés (Park, 2008).

La relación entre PI, innovación y desarrollo, desde una perspectiva empírica, ha sido documentada a distintas escalas e indistintamente del nivel de renta per cápita de las economías analizadas (Dosia \& Stiglitz, 2013; Higino Shneider, 2005). La naturaleza, efectividad e impacto de esa relación, en términos de innovación y desarrollo, depende de factores como la fortaleza institucional de los sistemas de PI y su adhesión a los marcos regulatorios internacionales como, por ejemplo, la adopción de acuerdos internacionales como los Trade-Related Intellectual Property Agreements o TRIP, por sus siglas en inglés (Acemoglu \& Akcigit, 2012; Dinopolus \& Segerstrom, 2010).

Por tanto, la exploración de la naturaleza empírica de la relación entre PI, innovación y desarrollo 
supone dos cosas: a) asumir que esa relación puede ser medida a partir de un conjunto de variables, y b) que esa medición es deseable por sí misma para mejorar las políticas públicas de CTI. Lo anterior implica comprender la relevancia de las capacidades tecnológicas de los sistemas productivos como una expresión de la calidad de articulación de los SI en las distintas economías (Fagerberg \& Srholec, 2008; Lundvall, 2007). ${ }^{6}$

Una segunda cuestión por dilucidar es la de los SI como marco general de análisis. En términos más rigurosos, los SI pueden ser entendidos como el "arreglo" de instituciones, actores y sus interacciones relativas a los procesos de producción, almacenamiento, transferencia y uso del conocimiento para la creación de valor en los sistemas económicos (Metcalfe \& Ramlogan, 2008). Precisamente una de las funciones de un sistema de innovación es proveer las reglas de juego que, en materia de propiedad intelectual, pauten el comportamiento esperado de los agentes económicos (Edquist, 2005). ${ }^{7}$ Por igual, una de las funciones de la PI como parte de un SI es asegurar que los resultados del esfuerzo innovador de las firmas o de otros agentes precursores del sistema, medido por ejemplo en gasto en investigación y desarrollo $(\mathrm{I}+\mathrm{D})$, se vea protegido por un determinado período de tiempo que les permita recuperar la inversión y, de ese modo, crear valor en términos de retorno financiero (Dosia \& Stiglitz, 2013; Scotchmer, 2004).

6. Desde el punto de vista epistemológico, la cuestión de medir la relación entre PI y desarrollo implica que las variables a ser analizadas pueden ser identificadas y definidas de forma relativamente independiente y que tales variables interactúan para la creación de valor, tanto a nivel de firmas individuales o por sector, como a nivel agregado dentro del sistema económico (Bollen, Vergauwen, \& Schnieders, 2005; Dosia \& Stiglitz, 2013).

7. Los SI pueden tener distintas escalas y diferente alcance: se puede hablar de sistemas regionales, nacionales y locales de innovación, así como de sistemas sectoriales de innovación relativos a un sector o a una actividad económica en cierta escala (Geels, 2004; Meeus \& Oerlemans, 2005).
Finalmente, el efecto agregado de la creación de valor mediante el uso del conocimiento en un SI constituye un motor del desarrollo medido en términos de la creación de riqueza y bienestar. Lo anterior se puede aproximar mediante un indicador de amplio uso, como la renta per cápita que, a pesar de sus limitaciones y críticas, ofrece una relación generalmente aceptada entre crecimiento económico, fuerza laboral y bienestar social; relación que ya ha sido previamente explorada en cuanto a la política científica y tecnológica, así como con respecto a la relación entre PI, innovación y desarrollo.

\section{Metodología}

En términos metodológicos, el punto de partida ha sido la construcción de un panel de datos con información correspondiente al período comprendido entre 2005 y 2011, por ser un ciclo con información bastante completa desde el punto de vista de los datos de las variables. El análisis de datos de panel en la literatura sobre propiedad intelectual se ha consolidado a lo largo de la última década; se incluyen como ejemplo algunos de los trabajos citados a lo largo del presente análisis (Branstetter, Fisman \& Foley, 2006; Higino Shneider, 2005).

Los datos de panel ofrecen claras ventajas metodológicas para el análisis de la PI sobre otros métodos como el análisis de series temporales. Algunas de esas ventajas son: un mejor control de la heterogeneidad individual, asumiendo precisamente que tanto las firmas como los países (nivel agregado de análisis) son heterogéneos; más información y variabilidad $y$, por consiguiente, menos colinealidad entre los datos y las variables; la detección de efectos entre las variables que usualmente pueden pasar desapercibidos, entre otras ventajas analíticas (Baltagi, 2005). Esto es especialmente útil cuando se analiza la PI relacionada con dinámicas 
complejas, como la innovación, el cambio tecnológico y el crecimiento (Acemoglu \& Akcigit, 2012; Dinopolus \& Segerstrom, 2010; Kim et al., 2012).

Para este análisis, se generó un panel de datos compuesto por 41 variables tomadas principalmente del: a) Reporte Mundial de Competitividad (WEF, 2009); b) la base de datos de los Indicadores de Desarrollo del Banco Mundial (World-Bank, 2012), y c) datos sobre patentes y marcas de la Oficina de Comercio, Patentes y Marcas de los Estados Unidos (USPTO, 2012). En el anexo 1 se encuentra la relación de variables utilizadas en el panel de datos.
Con la finalidad de simplificar el análisis y reducir el número de variables, tal como se indicó previamente, se llevó a cabo un análisis factorial mediante el método de componentes principales, un procedimiento recomendado y utilizado con éxito en los estudios de capital intelectual (Bollen et al., 2005; Bontis, 1998). El análisis factorial permitió reducir el número de variables en los factores que más eficientemente pueden explicar la varianza de los datos del panel construido; estos factores se presentan a continuación en la tabla 1.

\section{Tabla 1. Factores generados mediante el método de componentes principales}

\begin{tabular}{|c|c|c|c|c|}
\hline Símbolos & Variable & Fuente & $\begin{array}{l}\text { Porcentaje individual de la } \\
\text { varianza explicada }\end{array}$ & Año de referencia \\
\hline$\Upsilon$ & PIB per cápita a precios corrientes & $\begin{array}{l}\text { World Development Indicators/Banco } \\
\text { Mundial. }\end{array}$ & $\mathrm{NA}^{*}$ & 2011 \\
\hline$\delta$ & $\begin{array}{l}\text { Etapa de desarrollo (cinco etapas incluyendo las de } \\
\text { transición) }\end{array}$ & $\begin{array}{l}\text { Global Competitiveness Report/World } \\
\text { Economic Forum }\end{array}$ & $\mathrm{NA}^{*}$ & 2011 \\
\hline$\rho$ & Patentes otorgadas en la USTPO & $\begin{array}{l}\text { Oficinas de Comercio, Patentes y Marcas } \\
\text { de los Estados Unidos }\end{array}$ & $\mathrm{NA}^{*}$ & 2011 \\
\hline$\beta 1$ & $\begin{array}{l}\text { Porcentaje (\%) de fuerza laboral con educación } \\
\text { secundaria }\end{array}$ & $\begin{array}{l}\text { World Development Indicators/Banco } \\
\text { Mundial. }\end{array}$ & 0.94 & 2011 \\
\hline$\beta 2$ & $\begin{array}{l}\text { Porcentaje (\%) de fuerza laboral con educación } \\
\text { universitaria }\end{array}$ & $\begin{array}{l}\text { World Development Indicators/Banco } \\
\text { Mundial. }\end{array}$ & 0.92 & 2011 \\
\hline$\beta 3$ & $\begin{array}{l}\text { Porcentaje (\%) de fuerza laboral con educación } \\
\text { primaria }\end{array}$ & $\begin{array}{l}\text { World Development Indicators/Banco } \\
\text { Mundial. }\end{array}$ & 0.90 & 2011 \\
\hline$\beta 4$ & Índice de innovación WEF & $\begin{array}{l}\text { Global Competitiveness Report/World } \\
\text { Economic Forum }\end{array}$ & 0.90 & 2005 \\
\hline$\beta 5$ & Grado de protección de la PI WEF & $\begin{array}{l}\text { Global Competitiveness Report/World } \\
\text { Economic Forum }\end{array}$ & 0.81 & 2005 \\
\hline$\beta 6$ & Grado de apertura económica & $\begin{array}{l}\text { World Development Indicators/Banco } \\
\text { Mundial. }\end{array}$ & 0.74 & 2011 \\
\hline$\beta 7$ & $\begin{array}{l}\text { Investigación \& Desarrollo (I+D) total \% como } \\
\text { del PIB }\end{array}$ & $\begin{array}{l}\text { World Development Indicators/Banco } \\
\text { Mundial. }\end{array}$ & 0.70 & 2011 \\
\hline$\beta 8$ & Fortaleza del sistema de PI & $\begin{array}{l}\text { Global Competitiveness Report/World } \\
\text { Economic Forum }\end{array}$ & 0.70 & 2011 \\
\hline$\beta 9$ & $\begin{array}{l}\text { Inversión Extranjera Directa (IED) como \% del } \\
\text { PIB }\end{array}$ & $\begin{array}{l}\text { World Development Indicators/Banco } \\
\text { Mundial. }\end{array}$ & 0.65 & 2011 \\
\hline$\beta 10$ & Publicaciones totales en C\&T & $\begin{array}{l}\text { World Development Indicators/Banco } \\
\text { Mundial. }\end{array}$ & 0.48 & 2011 \\
\hline
\end{tabular}

${ }^{*} \mathrm{NA}=$ no aplica. Variables no sometidas al proceso de reducción factorial mediante componentes principales

Fuente: World Economic Forum (2009); World Bank (2012); USPTO (2012). 
Tabla 2. Resumen de las especificaciones generales de los modelos de regresión

\begin{tabular}{|c|c|c|c|c|}
\hline Estimadores & Modelo 1 & Modelo 2 & Modelo 3 & Modelo 4 \\
\hline $\mathrm{R}$ & 0.885 & 0.775 & 0.870 & 0.841 \\
\hline $\mathrm{R}$ cuadrado & 0.783 & 0.600 & 0.757 & 0.707 \\
\hline R cuadrado corregida & 0.777 & 0.596 & 0.751 & 0.704 \\
\hline Error típico de la estimación & 0.68328 & 0.95799 & 0.19493 & 0.21321 \\
\hline \multicolumn{5}{|c|}{ ANOVA } \\
\hline \multicolumn{5}{|l|}{ Regresión } \\
\hline Suma de cuadrados & 429.434 & 478.433 & 30.226 & 38.073 \\
\hline $\mathrm{gl}$ & 7 & 4 & 6 & 4 \\
\hline Media cuadrática & 61.348 & 119.608 & 2.038 & 9.518 \\
\hline $\mathrm{F}$ & 131.401 & 130.327 & 132.583 & 209.374 \\
\hline Sig. & 0.000 & 0.000 & 0.000 & 0.000 \\
\hline \multicolumn{5}{|l|}{ Residual } \\
\hline Suma de cuadrados & 119.053 & 318.461 & 9.727 & 15.775 \\
\hline $\mathrm{gl}$ & 255 & 347 & 256 & 347 \\
\hline \multirow[t]{4}{*}{ Media cuadrática } & & & & 0.045 \\
\hline & 0.467 & & & \\
\hline & 0.918 & & & \\
\hline & 0.038 & & & \\
\hline \multicolumn{5}{|l|}{ Total } \\
\hline Suma de cuadrados & 548.487 & 796.893 & 39.954 & 53.848 \\
\hline gl & 262 & 351 & 262 & 351 \\
\hline
\end{tabular}

Tal como se puede apreciar en la tabla 1, las 41 variables iniciales fueron reducidas a 10 factores que explican de manera conjunta poco más de dos tercios de la varianza total de los datos del panel. Las tres primeras variables: el PIB per cápita a precios corrientes $(\gamma)$, las etapas de desarrollo de las distintas economías analizadas $(\delta)$ y las patentes otorgadas $(\rho)$ se consideran variables explicadas o dependientes en los modelos de regresión generados. El índice de innovación $\left(\beta_{4}\right)$ será, al mismo tiempo, variable explicativa (en el caso de los modelos sobre PIB per cápita) y variable explicada en los modelos correspondientes. ${ }^{8}$

8. En la construcción del panel de datos en lugar de utilizar la variable INB per cápita se optó por utilizar la medida más convencional, relativa al PIB per cápita, por estar mejor documentada con anterioridad al año 2010 en bases de datos internacionales, a pesar de que en 2010, para el Informe de Desarrollo
Una vez obtenidos los factores o las variables de mayor peso en la explicación de la varianza, siguiendo a otros autores (Acemoglu \& Akcigit, 2012; Baltagi, 2005; Kim et al., 2012), se procedió a realizar una transformación de logaritmos naturales o LN, con el propósito de estandarizar y normalizar el comportamiento de los factores y de las variables explicadas. Con los resultados de la transformación logarítmica se pasó a estimar las regresiones mediante el método de mínimos cuadrados ordinarios, siguiendo el procedimiento Stepwise o paso a paso, que permite introducir o eliminar variables dependiendo de su capacidad discriminatoria, lo que hace progresivamente más

Humano de ese año, se optó por utilizar el INB para medir el estándar de vida de la población (UNDP, 2010). 
restrictivo cada modelo estimado (Silhavy, Silhavy \& Prokopova, 2017). Se utilizó el software estadístico SPSS@, en su versión 20.0.

\section{Resultados}

Se estimaron cuatro grandes modelos con niveles progresivos de restricción. El primero y el segundo se estimaron teniendo como variable dependiente la renta per cápita, y en el tercero y el cuarto como variable dependiente se utilizó el índice de innovación estimado por el World Economic Forum, como variable normalizada. Las variables independientes para los cuatro modelos estimados fueron los 10 factores presentados en la tabla 1 . A continuación, el resumen de los cuatro modelos se presenta en la tabla 2.
Lo primero que destaca en la tabla 2 es el buen ajuste y la capacidad explicativa de los cuatro modelos estimados. La varianza de los datos de panel ( $\mathrm{R}, \mathrm{R}^{2}$ ajustada) es de un $78 \%$ en el modelo 1 y de un $60 \%$ en el modelo 2 . El modelo 3 explica cerca del $76 \%$ de la varianza $(75.7 \%)$ y el modelo 4 cerca del $71 \%(70.7 \%)$. Estos resultados son muy buenos en cualquier escenario analítico y hablan bien de la robustez de las estimaciones, resaltando, además, que los cuatro modelos fueron significativos a 5\% de nivel de confianza (Chen \& Puttitanun, 2005; Kim et al., 2012).

En la tabla 3 se aprecian los resultados para los modelos 1 y 2 , que tienen como variable dependiente el PIB per cápita $(\gamma)$.

Tabla 3. Modelos de regresión PIB per cápita

\begin{tabular}{|c|c|c|c|c|c|c|c|c|c|c|c|}
\hline \multirow{3}{*}{ Símbolos } & \multirow{3}{*}{ Factor/variable } & \multicolumn{5}{|c|}{ Modelo 1} & \multicolumn{5}{|c|}{ Modelo 2} \\
\hline & & \multicolumn{2}{|c|}{$\begin{array}{l}\text { Coeficientes no } \\
\text { estandarizados }\end{array}$} & \multirow{2}{*}{$\begin{array}{c}\begin{array}{c}\text { Coeficientes } \\
\text { tipificados }\end{array} \\
\text { Beta }\end{array}$} & \multirow[t]{2}{*}{$\mathrm{t}$} & \multirow{2}{*}{ Sig. } & \multicolumn{2}{|c|}{$\begin{array}{l}\text { Coeficientes no } \\
\text { estandarizados }\end{array}$} & \multirow{2}{*}{$\begin{array}{c}\begin{array}{c}\text { Coeficientes } \\
\text { tipificados }\end{array} \\
\text { Beta }\end{array}$} & \multirow[t]{2}{*}{$\mathrm{t}$} & \multirow{2}{*}{ Sig. } \\
\hline & & $\mathrm{B}$ & Error tip. & & & & $\mathrm{B}$ & Error tip. & & & \\
\hline$\bigotimes$ & Constante & 0.413 & 0.667 & & 0.619 & 0.536 & 3.994 & 0.347 & & 11.380 & 0.000 \\
\hline$\otimes 1$ & $\begin{array}{l}\text { Porcentaje }(\%) \text { de fuerza laboral con } \\
\text { educación secundaria }\end{array}$ & 0.418 & 0.072 & 0.194 & 5.772 & 0.000 & & & & & \\
\hline$\bigotimes 2$ & $\begin{array}{l}\text { Porcentaje }(\%) \text { de fuerza laboral con } \\
\text { educación universitaria }\end{array}$ & 0.168 & 0.083 & 0.079 & 2.017 & 0.045 & & & & & \\
\hline 邓3 & $\begin{array}{l}\text { Porcentaje }(\%) \text { de fuerza laboral con } \\
\text { educación primaria }\end{array}$ & 0.227 & 0.071 & 0.116 & 3.189 & 0.002 & & & & & \\
\hline$\bigotimes 4$ & Índice de innovación WEF & 1.657 & 0.219 & 0.447 & 7.562 & 0.000 & & & & & \\
\hline 冈5 & Grado de protección de la PI WEF & 2.159 & 0.238 & 0.470 & 9.086 & 0.000 & 2.551 & 0.229 & 0.536 & 11.146 & 0.000 \\
\hline$\nabla 6$ & Grado de apertura económica & 0.263 & 0.085 & 0.093 & 3.079 & 0.002 & & & & & \\
\hline$\bigotimes 7$ & $\begin{array}{l}\text { Investigación \& Desarrollo (I+D) } \\
\text { total \% como del PIB }\end{array}$ & -0.124 & 0.060 & -0.087 & -2.073 & 0.039 & 0.101 & 0.066 & 0.070 & 1.543 & 0.124 \\
\hline$\bigotimes 8$ & Fortaleza del sistema de PI & & & & & & 0.538 & 0.256 & 0.094 & 2.098 & 0.037 \\
\hline$\nabla 9$ & $\begin{array}{l}\text { Inversión Extranjera Directa (IED) } \\
\text { como \% del PIB }\end{array}$ & & & & & & & & & & \\
\hline$\otimes 10$ & Publicaciones totales en C\&T & & & & & & 0.115 & 0.290 & 0.201 & 3.998 & 0.000 \\
\hline
\end{tabular}


En el caso del modelo 1, a un nivel del 5\% de confianza, prácticamente todas las variables son significativas. Resaltan, por su peso, las variables $\beta_{1}$ (porcentaje de la fuerza laboral con educación secundaria), $\beta_{4}$ (índice de innovación estimado por el WEF) y $\beta_{5}$ (grado de protección de la PI de acuerdo con el WEF). El factor $\beta_{2}$ (porcentaje de la educación laboral con educación universitaria) emerge de forma interesante en el modelo 1 y puede ser una indicación importante, cónsona con los hallazgos que resaltan el peso del capital humano con el desarrollo y, en particular, con la productividad de las firmas (Feitas Rodriguez, de Novaes Netto, Ramos, Alves Corrêa \& Giuliani, 2010).

El signo negativo del factor $\beta 7$ (investigación $\&$ desarrollo como parte del PIB) en el modelo 1 puede estar indicando un efecto selectivo, dada la escala de agregación de los datos del panel y la heterogeneidad de la información sobre los procesos de asignación del gasto en I+D (Bartelsman, Haltiwanger \& Scarpetta, 2013). En el caso del modelo 2, de mayor nivel de restricción que el modelo 1 , resalta por su peso las variables $\beta_{5}$ y se incluye, a diferencia del modelo 1 , la variable $\beta_{10}$ (publicaciones totales en $C \& T)$.

Los resultados muestran con claridad que entre $e l$ PIB per cápita, como variable aproximada de desarrollo, y los factores de propiedad intelectual e innovación existe una clara relación que no solo es significativa en términos estadísticos, sino también de tipo predictor. Esta relación es significativa independientemente del nivel de restricción del modelo. Lo anterior es consistente con hallazgos similares que relacionan los niveles de renta con sistemas fuertes de propiedad intelectual (Fagerberg $\&$ Srholec, 2008).

A pesar de la claridad y la consistencia del resultado, es importante acotar que los sistemas de protección de la propiedad intelectual no operan de forma aislada dentro del sistema económico, sino que son la expresión de un fuerte nivel de articulación y desarrollo institucional (Parto, 2008). Esto puede ser tomado como una lectura que resalta el peso del desarrollo institucional en las dinámicas de desarrollo económico y social (Lee \& Kim, 2009).

En distintos países de renta media la debilidad institucional parece ser un factor crítico, no solo desde la perspectiva del desarrollo, pues también afecta la consolidación de los sistemas de innovación que, por su sensibilidad, requieren de un adecuado sustento institucional y de un marco de políticas transparente (Nelson \& Nelson, 2002). Para concluir con las reflexiones derivadas del análisis de los dos modelos iniciales, basta con aseverar que en el contexto centroamericano y, por extensión, en la República Dominicana, una de las características de sus sistemas de innovación y de los marcos de política de CTI es precisamente su debilidad institucional y las fallas de coordinación interna (Padilla-Pérez \& Gaudin, 2014; Vasudeva, 2009).

En lo que respecta a la innovación y su importancia en las dinámicas de desarrollo, en la tabla 4 se aprecian los resultados para los modelos 3 y 4 , cuya variable dependiente es el índice de innovación; para identificarlo se utilizará el símbolo $\mathrm{t}$.

En el caso del modelo 3, se incorporaron 6 de los 10 factores, a un nivel del $5 \%$ de confianza; siendo significativos cuatro de los seis factores. Por orden de magnitud, destacan los factores $\beta_{5}$ (grado de protección de la PI), $\beta_{2}$ (porcentaje de la fuerza laboral con educación universitaria), $\beta_{7}$ (investigación $\&$ desarrollo como parte del PIB) y $\beta_{1}$ (porcentaje de la fuerza laboral con educación secundaria). En cuanto al modelo 4, se incorporaron cuatro de los diez factores. Destacan por su peso los factores $\beta_{5}$ (grado de protección de la PI), $\beta_{8}$ (fortaleza del sistema de PI), $\beta_{7}$ (investigación $\&$ desarrollo como parte del PIB) y $\beta_{10}$ (publicaciones totales en $\mathrm{C} \& \mathrm{~T}$ ). Coinciden en los modelos 3 y 4 los factores $\beta_{5}$ (grado de protección de la PI) y $\beta_{7}$ (investigación y desarrollo como $\%$ del PIB). 
Tabla 4. Modelos de regresión Índice de innovación

\begin{tabular}{|c|c|c|c|c|c|c|c|c|c|c|c|}
\hline \multirow{3}{*}{ Símbolos } & \multirow{3}{*}{ Factor/variable } & \multicolumn{5}{|c|}{ Modelo 3} & \multicolumn{5}{|c|}{ Modelo 4} \\
\hline & & \multicolumn{2}{|c|}{$\begin{array}{c}\text { Coeficientes no estan- } \\
\text { darizados }\end{array}$} & \multirow{2}{*}{$\begin{array}{c}\begin{array}{c}\text { Coeficientes } \\
\text { tipificados }\end{array} \\
\text { Beta }\end{array}$} & \multirow[t]{2}{*}{$\mathrm{t}$} & \multirow{2}{*}{ Sig. } & \multicolumn{2}{|c|}{$\begin{array}{l}\text { Coeficientes no } \\
\text { estandarizados }\end{array}$} & \multirow{2}{*}{$\begin{array}{c}\begin{array}{c}\text { Coeficientes } \\
\text { tipificados }\end{array} \\
\text { Beta }\end{array}$} & \multirow{2}{*}{$\mathrm{t}$} & \multirow{2}{*}{ Sig. } \\
\hline & & B & Error tip. & & & & B & Error tip. & & & \\
\hline$\otimes$ & Constante & -0.359 & 0.189 & & -1.900 & 0.059 & -0.141 & 0.077 & & -1.829 & 0.068 \\
\hline$\otimes 1$ & $\begin{array}{l}\text { Porcentaje (\%) de fuerza } \\
\text { laboral con educación se- } \\
\text { cundaria }\end{array}$ & 0.080 & 0.020 & 0.138 & 3.999 & 0.000 & & & & & \\
\hline$凶 2$ & $\begin{array}{l}\text { Porcentaje (\%) de fuerza } \\
\text { laboral con educación uni- } \\
\text { versitaria }\end{array}$ & 0.176 & 0.021 & 0.305 & 8.314 & 0.000 & & & & & \\
\hline$\nabla 3$ & $\begin{array}{l}\text { Porcentaje }(\%) \text { de fuerza } \\
\text { laboral con educación pri- } \\
\text { maria }\end{array}$ & -0.017 & 0.020 & -0.033 & -0.851 & 0.395 & & & & & \\
\hline$\nabla 4$ & Índice de innovación WEF & & & & & & & & & & \\
\hline$\triangle 5$ & $\begin{array}{l}\text { Grado de protección de la } \\
\text { PI WEF }\end{array}$ & 0.682 & 0.053 & 0.550 & 12.953 & 0.000 & 0.425 & 0.051 & 0.344 & 8.352 & 0.000 \\
\hline$\nabla 6$ & $\begin{array}{l}\text { Grado de apertura econó- } \\
\text { mica }\end{array}$ & -0.053 & 0.024 & -0.069 & -2.183 & 0.030 & & & & & \\
\hline$\otimes 7$ & $\begin{array}{l}\text { Investigación \& Desarrollo } \\
\text { (I+D) total \% como del PIB }\end{array}$ & 0.082 & 0.016 & 0.213 & 5.050 & 0.000 & 0.088 & 0.015 & 0.235 & 6.013 & 0.000 \\
\hline$凶 8$ & Fortaleza del sistema de PI & & & & & & 0.281 & 0.057 & 0.188 & 4.934 & 0.000 \\
\hline$凶 9$ & $\begin{array}{l}\text { Inversión Extranjera Directa } \\
\text { (IED) como \% del PIB }\end{array}$ & & & & & & & & & & \\
\hline$\otimes 10$ & $\begin{array}{l}\text { Publicaciones totales en } \\
\text { C\&T }\end{array}$ & & & & & & 0.040 & 0.006 & 0.269 & 6.266 & 0.000 \\
\hline
\end{tabular}

Con respecto a la PI, los resultados de los modelos 3 y 4 son consistentes con la literatura en el sentido de que, efectivamente, la fortaleza y eficacia de los sistemas de propiedad intelectual -medidos aquí por los factores $\beta_{5}$ y $\beta_{8}$, respectivamente- están relacionadas con la calidad y la eficiencia de los sistemas de innovación (Branstetter et al., 2006; Kim et al., 2012).

La relación anterior parece ser importante no solo en las economías desarrolladas, sino también en las economías en desarrollo (Chen \& Puttitanun, 2005). El resultado es consistente con hallazgos similares que relacionan los niveles de renta con sistemas fuertes de propiedad intelectual (Fagerberg $\&$ Srholec, 2008), así como con las estrategias de apuesta a la innovación como instrumentos para evitar la trampa de los ingresos medios, referida en la introducción (Griffith, 2011). Con base en este resultado, se puede validar lo dicho anteriormente, en el sentido de que los sistemas de propiedad intelectual no operan de forma aislada dentro del sistema económico, sino que son la expresión de un fuerte nivel de articulación y desarrollo institucional (Parto, 2008).

Otro resultado interesante se relaciona con la importancia de los factores de capital humano, los cuales muestran mayor importancia en los modelos 3 y 4 que en los modelos 1 y 2 . La razón puede estar en la importancia del capital humano para los procesos de innovación, lo que se expresa en requerimientos como la formación de personal especializado en los campos de las ciencias y las ingenierías. Tales requerimientos son elementos claves para explicar las dinámicas de producción de conocimiento inherentes a todo sistema de innovación (Fagerberg \& Srholec, 2008; Lytras, 2008). 
Vale afirmar que la literatura sobre el papel del capital humano en la productividad y en las capacidades tecnológicas, tanto a escala de las empresas como de las economías, escapa por su extensión a los límites del presente trabajo (Bartelsman et al., 2013; Cerulli, 2014). De todos modos, la relevancia del capital humano remite a la reflexión de fondo, de carácter seminal, sobre la importancia de la calidad de los sistemas educativos y su capacidad para ser inclusivos en términos sociales. Después de todo, es precisamente en los sistemas educativos y en su capacidad para ser inclusivos que se generan las capacidades que desarrollan el capital intelectual y sus posibilidades de creación de valor a escala país (Lin \& Edvinsson, 2010)

Tanto el modelo 3 como el número 4 comparten el factor $\beta_{7}$ (gasto total en $\mathrm{I}+\mathrm{D}$ como parte del PIB), lo que resalta la importancia de la actividad formal de producción de conocimiento orientada a los procesos de innovación; una cuestión más debatible en el contexto de países en desarrollo, debido al peso que tienen los procesos informales de aprendizaje e innovación (Chen \& Puttitanun, 2005; Metcalfe \& Ramlogan, 2008; Padilla-Pérez $\&$ Gaudin, 2014). Sin embargo, no cabe dudas de que el gasto racional en $\mathrm{I}+\mathrm{D}$, como aproximación del esfuerzo en materia de innovación, constituye un claro factor de anclaje para superar las limitaciones que impone la trampa del ingreso medio y para salir de ella (Doner \& Schneider, 2016).

En el modelo 4 se adiciona el factor $\beta_{10}$ (publicaciones totales en C\&T), que ya emergió en el modelo 2. Ciertamente, la producción científica es estimada por el número de publicaciones, lo que es un indicador importante de las capacidades científico-tecnológicas en una determinada economía (Fagerberg \& Srholec, 2008). De igual modo, la producción científica puede ser tomada como una expresión del grado de madurez de los sistemas de innovación, lo que no necesariamente refleja la situación de las relativamente pequeñas economías abiertas, como las centroamericanas y la dominicana (Padilla-Pérez \& Gaudin, 2014).

En lo referente al papel de la PI, existe evidencia, al menos para el caso dominicano, de que muestra un bajo nivel de conocimiento y apropiación, por parte de las empresas y de otros actores del SI, de las ventajas y oportunidades que los marcos institucionales vigentes de PI pueden ofrecerles para la creación de valor (Gómez-Valenzuela, 2014). Esta baja apropiación y relativo desconocimiento del potencial de la legislación de la PI puede estar relacionado de alguna forma con el también bajo esfuerzo formal en $\mathrm{I}+\mathrm{D}$ que realizan las empresas dominicanas y otros actores del SI (de Groote, 2015).

Finalmente, los resultados mostrados por los datos de panel ponen en evidencia que los factores de capital humano $\left(\beta_{1}, \beta_{2}, \beta_{3}\right)$, los sistemas de propiedad intelectual como expresión del grado de desarrollo institucional de las economías $\left(\beta_{5}, \beta_{8}\right)$ y el esfuerzo en producción de conocimiento $\left(\beta_{7}, \beta_{10}\right)$ muestran en su conjunto una lectura positiva acerca del papel que desempeńan en las dinámicas de crecimiento y desarrollo. Por igual, estos factores relacionan de manera clara la innovación con las dinámicas de creación de valor que dentro de un sistema de innovación impactan en el crecimiento económico (Verspagen, 2005).

\section{Conclusiones}

Las reflexiones presentadas a lo largo del presente trabajo, con base en los hallazgos de otros autores y, en particular, a partir de la evidencia empírica presentada en el apartado sobre resultados, muestran con claridad que la relación entre PI, innovación y desarrollo no es espuria y que, además, como se ha visto, es una relación bien documentada en la literatura especializada. Los distintos factores incorporados en las estimaciones muestran con toda claridad que, además del fuerte vínculo entre la PI, 
la innovación y el desarrollo, esa relación es fundamental para sustentar una dinámica de crecimiento y desarrollo a largo plazo, lo que parece sustancialmente importante para las economías de renta media. Las principales reflexiones documentadas en este trabajo se pueden resumir en los siguientes puntos:

- En primer lugar, la trampa de los ingresos medios parece afectar de manera sistémica a los países de América Latina, encontrándose entre estos países la República Dominicana. Los factores estructurales que determinan esta condición fueron superficialmente mencionados en la introducción, pero también el papel que puede desempeñar la inversión en innovación, así como en educación, como vías para evitar la referida trampa.

- En segundo término, la relación entre PI, innovación y desarrollo tiene la suficiente fuerza y consistencia como para aseverar que la formulación de políticas asertivas de CTI y el fortalecimiento de los sistemas de innovación se muestran como estrategias viables para estimular ciclos de crecimiento de largo plazo, que pueden evitar la trampa de los ingresos medios y promover la inclusión social.

- En tercer lugar, el fortalecimiento de los sistemas de propiedad intelectual, como pilar de los sistemas de innovación, no es una tarea menor. $\mathrm{Al}$ margen de cualquier consideración que pueda hacerse sobre el esfuerzo y las capacidades de $\mathrm{I}+\mathrm{D}$ de las empresas en economías como la dominicana, no cabe dudas que se requiere más divulgación y conocimiento sobre los sistemas de PI y su importancia.

- En cuarto lugar, los países como la República Dominicana y aquellos de América Latina y el Caribe atrapados en la trampa de los ingresos medios confrontan dos grandes desafíos ya documentados por otros autores (Doner \& Schneider, 2016): primero, la formulación de políticas que permitan relanzar la productividad con base en la formación del capital humano, el desarrollo tecnológico y la innovación; y segundo, abordar los desafíos anteriores en paralelo con la construcción y el fortalecimiento de las capacidades institucionales requeridas para articular sistemas de innovación funcionales. En este punto, el fortalecimiento de los sistemas de PI, como pilar de los sistemas de innovación, tomando en cuenta las peculiaridades de cada economía, constituye una tarea que tiene que ser acometida con persistencia.

- En quinto y último lugar, los sistemas de propiedad intelectual no pueden operar de manera independiente y al margen de los distintos actores e instituciones que forman parte de los sistemas de innovación. No solo son un pilar de los SI, sino que también tienen que responder a las necesidades de cada realidad y adaptarse a los requerimientos de crecimiento, desarrollo e inclusión de cada país.

Al menos para el caso de América Latina y el Caribe, parece clara la necesidad de contar con una nueva generación de políticas de CTI que se construyan desde una perspectiva integral, que permita incorporar a la política económica y fiscal como parte de un nuevo pacto por el desarrollo productivo y la inclusión social en la región. Esta parece ser una forma inteligente no solo de salir de la trampa de los ingresos medios, sino para construir las bases de un modelo de crecimiento y desarrollo que impacte en la calidad de vida de la población.

A modo de conclusión, el análisis de la relación entre PI, innovación y desarrollo está lejos de agotarse. Se requiere una mayor estilización metodológica para responder a cuestiones como el grado de dependencia entre el nivel de conocimiento de la PI y la inversión en $\mathrm{I}+\mathrm{D}$ en economías de renta media. Del mismo modo, falta por responder preguntas sobre los impulsores críticos de los procesos de innovación que permitan romper la trampa de 
los ingresos medios, cuestiones que apenas fueron referidos en el marco del presente estudio y que requieren de futuras exploraciones y análisis adicionales que sustenten una heurística complementaria al análisis de la relación entre PI, innovación y desarrollo, construida a partir de los hechos estilizados que definen la trampa de la renta o ingresos medios y a las economías afectadas.

\section{Referencias}

Acemoglu, D. \& Akcigit, U. (2012). Intellectual property rights policy, competition and innovation. Journal of the European Economic Association, 10 (1), 1-42. Doi: http://dx.doi.org/10.1111/j.1542 $-4774.2011 .01053 . x$

Arthur, W. B. (2009). The nature of technology. New York, NY. USA.: Free Press.

Baltagi, B. H. (2005). Econometric Analysis of Panel Data ( $3^{\mathrm{a}}$ ed.). West Sussex, England: John Wiley \& Sons, Ltd.

Bartelsman, E., Haltiwanger, J., \& Scarpetta, S. (2013). Cross-country differences in productivitiy: The role of allocation and selection. American Economic Review, 103(1), 305-334. Doi: http:// dx.doi.org/10.1257/aer.103.1.305

Bollen, L., Vergauwen, P., \& Schnieders, S. (2005). Linking intellectual capital and intellectual property to company performance. Management Decision, 43(9), 1161-1185. Doi: 10.1108/002517 40510626254

Bontis, N. (1998). Intellectual capital: An exploratory study that develops measures and models. Management Decision, 36(2), 63-76.

Branstetter, L. G., Fisman, R., \& Foley, C. F. (2006). Do stronger intellectual property rights increase international technology transfer? Empirical evidence from U. S. Firm-level panel data. The Quaterly Journal of Economics, 121(1), 321-349.
Casalet, M., \& Buenrostro, E. (2014). Central American regional integration in science, technology and innovation: A new challenge. International Review of Sociology: Revue Internationale de Sociologie, 24(2), 345-365. Doi: 10.1080/039 06701.2014 .933018

Casper, S. \& van Waarden, F. (2005). Introduction: Scanning literature on institutions, organizations and innovation. In F. van Waarden (Ed.), Innovation and institutions. A multidisciplinary review of the study of innovation systems. ( $1^{\mathrm{a}} \mathrm{ed}$.). Cheltenham, UK.: Edward Elgar.

Cerulli, G. (2014). The impact of technological capabilities on invention: An investigation based on country responsiveness scores. World Development, 59, 147-165. Doi: http://dx.doi.org/10.1016/j. worlddev.2014.01.019

Chen, Y. \& Puttitanun, T. (2005). Intellectual property rights and innovation in developing countries. Journal of Development Economics, (78), 474493. Doi: 10.1016/j.jdeveco.2004.11.005

de Groote, R. (2015). Apoyo al desarrollo y fortalecimiento del sistema nacional de innovación y competitividad. Banco Interamericano de Desarrollo (BID)/Ministerio de Economía, Planificación y Desarrollo (MEPyd). Santo Domingo, D. N. República Dominicana.

Dinopolus, E. \& Segerstrom, P. (2010). Intellectual property rights, multinational firms and economic growth. Journal of Develoment Economics, (92), 1327. Doi: 10.1016/j.jdeveco.2009.01.007

Dinopoulos, E. \& Kottaridi, C. (2008). The growth effects of national patent policies. Review of International Economics, 16(3), 499-515. Doi: 10.1111/j.1467-9396.2008.00742.x

Doner, R. F. \& Schneider, B. R. (2016). The middle-income trap: More politics than economics. World Politics, 68(4), 608-644. Doi: 10.1017/S004 3887116000095 
Dosia, G. \& Stiglitz, J. (2013). The role of intellectual property rights in the development process, with some lessons from developed countries: An introduction. Recuperado de http://hdl.handle. net/10419/89516

Dutrénit, G. (2012). Innovación para el desarrolo en América Latina: dónde estamos respecto a las masas críticas de capacidades. En: Innovación y desarrollo: retos para una sociedad global (pp. 173202). Madrid, España: Fundación Carolina.

Edquist, C. (2005). System of innovation: Perspectives and challenges. In J. Fagerberg \& R. R. Nelson (Eds.), The Oxford Handbook of Innovation. New York, USA.: Oxford University Press.

Eichengreen, B., Park, D. \& Shin, K. (2013). Growth Slowdowns redux: New evidence on the middle-income trap. Recuperado de http://www.nber. org/papers/w18673

Etzkowitz, H. \& Leydesdorff, L. (2000). The dynamics of innovation: From national systems and "mode 2" to a triple helix of university-industry-government relations. Research Policy, (29), 109-123.

Fagerberg, J. \& Srholec, M. (2008). National innovation systems, capabilities and economic development. Research Policy, (37), 1417-1435. Doi: 10. 1016/j.respol.2008.06.003

Feitas Rodriguez, C., de Novaes Netto, F., Ramos, C. R., Alves Corrêa, D., \& Giuliani, A. C. (2010). El capital humano como factor de innovación tecnológica: un estudio de caso de caso en una empresa globalizada. INVENIO, 12(24), 119-135.

Felipe, J. (2012). Tracking the middle-income trap: What is it, who is in it, and why? Part 1. Recuperado de https://www.adb.org/sites/default/files/publication/29847/economics-wp-307.pdf

Fu, X., Pietrobelli, C., \& Soete, L. (2010). The role of foreign technology and indigenous innovation in emerging economies: Technological change and catching up. Recuperado de http://services.iadb. org/wmsfiles/products/Publications/35337751. pdf

Geels, F. W. (2004). From sectoral systems of innovation to socio-technical systems. Insights about dynamics and change from sociology and institutional theory. Research Policy, (33), 897-920.

Gómez-Valenzuela, V. (2014). Análisis del capital intelectual de empresas dominicanas: la importancia de las ideas y del conocimiento. Recuperado de: http://portalcdc.org/index.php?option=com_docman \&task=doc_download $\&$ gid $=397 \&$ Itemi$\mathrm{d}=203 \&$ lang=es

Gómez-Valenzuela, V. (2015). Analysis of preference of incentives to innovation of Dominican manufacturing and service firms. Journal of Technology Management \& Innovation, 10(2), 93-116. doi:10.4067/S0718-27242015000200007

Gómez-Valenzuela, V. (2016). Evidencia del efecto del capital intelectual en el desempeño de empresas de la República Dominicana. Ciencia y Sociedad, 41(4), 823-868.

Gould, D. M. \& Gruben, W. C. (1996). The role of intellectual property rights in economic growth. Journal of Development Economics, 48(2), 323350. Doi: https://doi.org/10.1016/0304-3878 (95)00039-9

Griffith, B. (2011). Middle-income trap. In R. Nallari, S. Yusuf, B. Griffith, \& R. Bhattacharya (Eds.), Frontier in Development Policy. Washington, D. C.: The World Bank.

Higino Shneider, P. (2005). International trade, economic growth and intellectual property rights: A panel data study of developed and developing countries. Journal of Development Economics, (78), 529-547. Doi: 10.1016/j.jdeveco.2004.09.001

Kim, Y. K., Lee, K., Park, W. G., \& Choo, K. (2012). Appropriate intellectual property protection and 
economic growth in countries at different levels of development. Research Policy, (41), 358-375. doi:10.1016/j.respol.2011.09.003

Lee, K., \& Kim, B.-Y. (2009). Both institutions and policies matter but differently for different income groups of countries: Determinants of long-run economic growth revisited. World Development, 37(3), 533-549. Doi: 10.1016/j. worlddev.2008.07.004

Lin, C. Y.-Y., \& Edvinsson, L. (2010). National intellectual capital a comparison of 40 countries. Recuperado de http://www.columbia.edu/cgi-bin/ cul/resolve?clio8574114

Lundvall, B. A. (2007). National innovation systems: Analytical concept and development tool. Industry and Innovation, 14(1), 95-119.

Lytras, M. D. (2008). Knowledge management strategies: A handbook of applied technologies. Hershey: IGI Pub.

Meeus, M. \& Oerlemans, L. (2005). National innovation systems Innovation and Institutions. A multidisciplinary review of the study of innovation systems (Casper, Steven; van Waarden, Frans eds.). Cheltenham, U. K.: Edward Elgar.

Metcalfe, J. S. \& Ramlogan, R. (2008). Innovation systems and the competitive process in developing countries. Quarterly Review of Economics and Finance, 48(2), 433-446. Doi: http://dx.doi.org/10.1016/j.qref.2006.12.021

Nallari, R., Yusuf, S., Griffith, B., \& Bhattacharya, R. (2011). Frontiers in development policy. Washisgton, D. C.: The International Bank for Reconstruction and Development/The World Bank.

Navarro, J. C., Benavente, J. M., \& Crespi, G. (2016). The new imperative of innovation: Policy perspectives for Latin America and the Caribbean. Recuperado de https://publications.iadb.org/
bitstream/handle/11319/7417/CTI-MONThe-New-Imperative-of-Innovation-PolicyPerspectives-for-LAC.pdf?sequence $=1$

Nelson, R. R. \& Nelson, K. (2002). Technology, institutions, and innovation systems. Research Policy, 31(2), 265-272. Doi: http://dx.doi. org/10.1016/s0048-7333(01)00140-8

Padilla-Pérez, R. \& Gaudin, Y. (2014). Science, technology and innovation policies in small and developing economies: The case of Central America. Research Policy, (43), 749-759. Doi: http://dx.doi. org/10.1016/j.respol.2013.10.011

Park, W. G. (2002). Institutions and incentives for $R \& D$ : Implications for LAC economies. Recuperado de http://www.american.edu/cas/faculty/wgpark/ upload/RD_LatinAm.pdf

Park, W. G. (2008). International patent protection: 1960-2005. Research Policy, (37), 761-766. Doi: 10.1016/j.respol.2008.01.006

Parto, S. (2008). Innovation and economic activity: An institutional analysis of the role of the clusters in industrializing economies. Journal of Economic Issues, 42(4), 1005-1030.

Scotchmer, S. (2004). Innovation and incentives. Cambridge, MA., United States: MIT Press Book.

Silhavy, R., Silhavy, P., \& Prokopova, Z. (2017). Analysis and selection of a regression model for the Use Case Points method using a stepwise approach. The Journal of Systems and Software, (125), 1-14. Doi: http://dx.doi.org/10.1016/j.jss.2016.11.029

UNDP. (2010). Human development report. The real wealth of nations: Pathways to human development. Recuperado de: http://hdr.undp.org/sites/ default/files/reports/270/hdr_2010_en_complete_reprint.pdf

USPTO. (2012). USPTO Statistics. Recuperado de http://www.uspto.gov/about/stats/index.jsp 
Vasudeva, G. (2009). How national institutions influence technology policies and firms' knowledge-building strategies: A study of fuel cell innovation across industrialized countries. Research Policy, (38), 1248-1259. Doi: 10.1016/j. respol.2009.05.006

Vergauwen, P., Bollen, L., \& Oirbans, E. (2007). Intellectual capital disclosure and intangible value drivers: An empirical study. Management Decision, 45(7), 1163-1180. Doi: $10.1108 / 00251740710773961$

Verspagen, B. (2005). Innovation and economic growth. The Oxford Handbook of Innovation. Oxford: Oxford University Press.
WEF. (2009). The global competitiveness report 2009-2010. Recuperado de http://www3.weforum.org/docs/WEF_GlobalCompe titivenessReport_2009-10.pdf

WIPO. (2004). Intellectual Property Handbook. Recuperado de http://www.wipo.int/edocs/pubdocs/en/intproperty/489/wipo_pub_489.pdf

World-Bank. (2012). World development indicators. Recuperado de http://web.worldbank.org/ website/external/countries

World-Bank. (2017). The Dominican Republic. Recuperado de http://www.worldbank.org/en/ country/dominicanrepublic

\section{Anexo 1. Variable y fuentes utilizadas}

\begin{tabular}{|c|c|c|c|c|}
\hline Num. & Variables codes & Variable's name & Reference Years & Source \\
\hline 1 & stagedevelop & Stage of development & $2009-2010$ & $\begin{array}{l}\text { Global Competitiveness } \\
\text { Report }\end{array}$ \\
\hline 2 & $\begin{array}{l}\text { patentgrante- } \\
\text { dUSPTO }\end{array}$ & $\begin{array}{l}\text { Patent granted by the } \\
\text { United States Patent and } \\
\text { Trademark Office }\end{array}$ & 2005-2009 & $\begin{array}{l}\text { United States Patent and } \\
\text { Trademark Office }\end{array}$ \\
\hline 3 & $\begin{array}{l}\text { r\&dtotal\%G- } \\
\text { DPEUM }\end{array}$ & $\begin{array}{l}\text { Research \& Development } \\
\text { as part of the GDP }\end{array}$ & 2011 & $\begin{array}{l}\text { Development Indicators, } \\
\text { World Bank }\end{array}$ \\
\hline 4 & $\begin{array}{l}\text { businessr\&d\%to- } \\
\text { tr\&d }\end{array}$ & $\begin{array}{l}\text { Company spending on } \\
\text { R\&D scores }\end{array}$ & 2011 & $\begin{array}{l}\text { Global Competitiveness } \\
\text { Report }\end{array}$ \\
\hline 5 & techreadinessWef & $\begin{array}{l}\text { Technological readiness } \\
\text { scores }\end{array}$ & 2009 & $\begin{array}{l}\text { Global Competitiveness } \\
\text { Report }\end{array}$ \\
\hline 6 & $\begin{array}{l}\text { innovationsco- } \\
\text { resWef }\end{array}$ & $\begin{array}{l}\text { Innovation scores in the } \\
\text { Global Competitiveness } \\
\text { Report }\end{array}$ & $2009-2010$ & $\begin{array}{l}\text { Global Competitiveness } \\
\text { Report }\end{array}$ \\
\hline 7 & ipprotectionWef & $\begin{array}{l}\text { Intellectual property pro- } \\
\text { tection scores }\end{array}$ & 2010 & $\begin{array}{l}\text { Global Competitiveness } \\
\text { Report }\end{array}$ \\
\hline 8 & patentrightindex & $\begin{array}{l}\text { International patent rights } \\
\text { index }\end{array}$ & 2008-2009 & $\begin{array}{l}\text { International Property Rights } \\
\text { Index }\end{array}$ \\
\hline 9 & $\begin{array}{l}\text { exportgYscurren- } \\
\text { tUS }\end{array}$ & $\begin{array}{l}\text { Exports of goods and servi- } \\
\text { ces (current US\$) }\end{array}$ & $2005-2011$ & $\begin{array}{l}\text { Development Indicators, } \\
\text { World Bank }\end{array}$ \\
\hline 10 & exports\%GDP & $\begin{array}{l}\text { Exports of goods and servi- } \\
\text { ces (\% of GDP) }\end{array}$ & $2005-2011$ & $\begin{array}{l}\text { Development Indicators, } \\
\text { World Bank }\end{array}$ \\
\hline
\end{tabular}


11 exportsannual\%- Exports of goods and servi- 2005-2011 growth ces (annual \% growth)

12 gdpcurrentUS GDP (current US\$)

13 gDPgrowthann\% GDP growth (annual \%)

14

$$
\text { gDPperca- }
$$
pitaconstan-

GDP per capita (constant 2000 US\$)

t2000US\$

15 gDPpercapitacurrentUS\$

16 gDPpercapitagrowthann \%

\section{grosssavings $\% \mathrm{G}$ -} DP

\section{hightechex-} ports\%ofmanufactexports importgYscurrentUS

import-

sofgoods\&services\%GDP
GDP per capita (current 2005-2011 US\$)

GDP per capita growth (annual \%)

Gross savings (\% of GDP) 2005-2011

High-technology exports

(\% of manufactured exports)

Imports of goods and servi- 2005-2011 ces (current US\$)

Imports of goods and servi- 2005-2011 ces (\% of GDP)

Imports of goods and servi- 2005-2011 vicesann\%growth ces (annual \% growth)

openness Trade openness

laborforceprime-

Labor force with primary ducation\%oftotal education (\% of total) laborforcesecondeducation\%ofLabor force with secondary total

5 laborforcetertiar- Labor force with tertiary yeducation \%total education (\% of total) patenappnonresi- Patent applications, dents nonresidents patenappresidents

Patent applications, residents primarycompletrattot
Primary completion rate, 2005-2011 total (\% of relevant age group) 2005-2011

2005-2011

2005-2011

2005-2011

2005-2011

2005-2011 2005-2011

Development Indicators, World Bank

Development Indicators, World Bank

Development Indicators, World Bank

Development Indicators, World Bank

Development Indicators, World Bank

Development Indicators, World Bank

Development Indicators, World Bank

Development Indicators, World Bank

Development Indicators, World Bank

Development Indicators, World Bank

Development Indicators, World Bank

Development Indicators, World Bank

Development Indicators, World Bank

Development Indicators, World Bank

Development Indicators, World Bank

Development Indicators, World Bank

2005-2011 Development Indicators, World Bank

Development Indicators, World Bank 


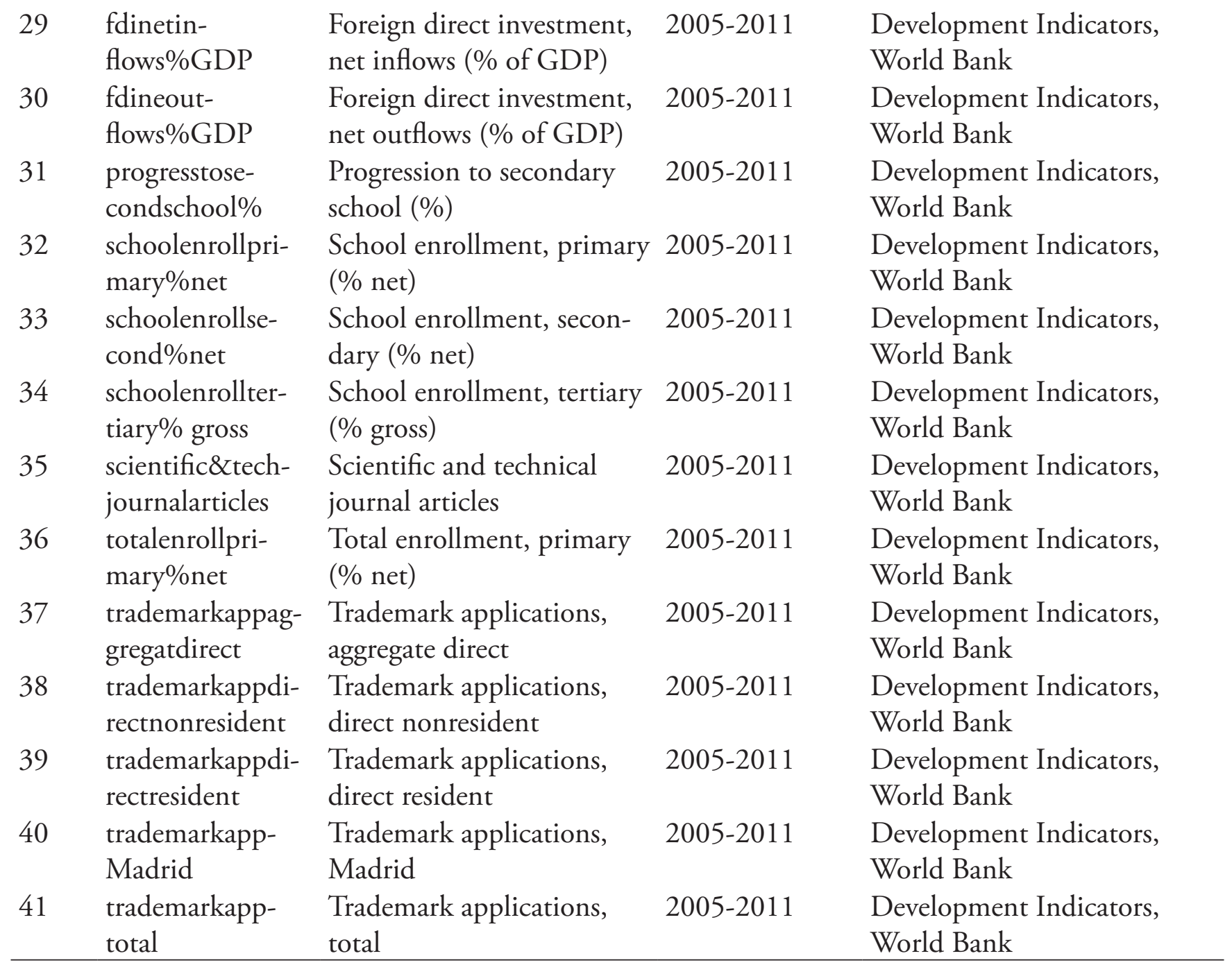

\section{Datos de filiación}

Víctor Gómez-Valenzuela. Es egresado de la Universidad Autónoma de Santo Domingo (UASD), donde estudió Antropología y se graduó con honores académicos; Master of Arts en Estudios Sociales de la Ciencia y la Tecnología por la Universidad de Maastricht de los Países Bajos y la European Inter-University Association on Science \& Technology. Además posee maestría Interuniversitaria en Economía y Gestión de la Innovación por las universidades Autónoma de Madrid, Complutense de Madrid y Politécnica de Madrid, así como un doctorado Interuniversitario en Ciencias Económicas por las referidas universidades. Líneas de investigación: capital humano; la innovación industrial; desarrollo de la propiedad industrial. Correo electrónico: victor.gomez@intec.edu.do 